DOI: 10.2478/ep-2018-0010

\author{
Zbigniew Mikrut \\ AGH University of Science and Technology \\ Kraków, Poland \\ Michał Widacki \\ University of Silesia in Katowice \\ Katowice, Poland \\ Jan Widacki*
}

\title{
Experiments in Using Face Temperature Changes as an Indicator in Instrumental Detection of Deception
}

Эксперименты с использовании изменений температуры лица

в качестве индикатора в инструментальной детекции лжи

Key words: Face temperature changes and selection of deception, infrared camera in detection of deception

\section{Introduction}

Variations of face temperature are certainly a good indicator of emotional changes, which is why attempts at using them in the detection of deception are being made.[1] Such variations may be observed with the use of an infrared camera, which implies

*jan.widacki@gmail.com 
remote sensing and removes the need to put any sensors on the body of the subject and makes the method potentially attractive for detection of deception. Theoretically, it can be applied without making the subject aware, and even more so without the subject's consent, which may material for its use for the purposes of police and/ or special units.

In infrared imaging changes of facial temperature are manifested by changes of colours of its various areas. The imaging can deploy full range of colours, from blue to dark red.

There are a number of potential methods for transforming the changes of colour spots into a viable graphic chart. One was patented in the US and the UK,[2] and another one in the UK.[3] We made such first successful attempts at such processing of the changing colour patterns into a graphic chart and described them ourselves in 2016.[4]

With this problem solved, another one arrived. Even minor movements of the subject's head, so small they would be insignificant and would in no way obstruct a classical polygraph examination, interrupted observation of the fragments of the face selected for monitoring, as the observed area disappeared from the field of view of the camera. This entailed the need to design an algorithm that would let the camera follow a selected area of the face in a continuous manner.

The implementation of an appropriate algorithm and the results of the test are described in section 2 below.

Section 3 describes the results of observations of temperature changes for multiple rectangular areas ("windows") located in various parts of the face that were later compared with the polygraph record consisting in the analysis of breathing functions, the work of the cardio-vascular system (the pulse and the relative fluctuations in blood pressure), and the galvanic skin (electrodermal) response. Another phenomenon studied was the impact of the size and shape of the selected "window" on the results achieved.

Recordings of the reactions of the subject to polygraph test using the Utah Zone Comparative Test (Utah ZCT) [...] technique were used to test the algorithm for detection of facial temperature changes with a relatively remote infrared camera, i.e. approx. $200 \mathrm{~cm} / 78 \mathrm{in}$. away from the face of the subject (see section 3 ). 


\section{Elimination of the impact of minor movements of the face of the subject}

To achieve this goal, the researchers first had to define the "windows", that is rectangular fields on the face of the subject, within which the image was to be analysed.[5] It was essential that the infrared camera continuously tracks them in the successive frames of the film. The selection of an area had to account for three criteria simultaneously.

First, it had to contain objects with contrasted colours. Secondly, it had to be positioned in an area with largest temperature changes, that is in the vicinity of major blood vessels. Thirdly and finally, the objects it covered should be invariable in their shape and not change their position in relation to the windows, and even more so to the camera itself, which means that these should be the areas that change least due to the activity of facial muscles. With the criteria above in mind, the lower section of the nose was selected for the reference window (benchmark). This required setting the camera so as to give it a view of the nostrils. The reference window was surrounded with a border, within which the pattern was sought on the successive frames of the film. The size of the border defines the scope of permitted movements of the face. The reference was shifted within thus defined border. The correlation coefficient was calculated for every new position:

$$
\gamma(u, v)=\frac{\sum_{x, y}\left[f(x, y)-\bar{f}_{u, v}\right][t(x-u, y-v)-\bar{t}]}{\left\{\sum_{x, y}\left[f(x, y)-\bar{f}_{u, v}\right]^{2} \sum_{x, y}[t(x-u, y-v)-\bar{t}]^{2}\right\}^{0.5}}
$$

where $f$ is the image of the frame, for which the $t$ benchmark is sought, $f_{u, v}$ is the average value of the image $f(x, y)$ in the area below the reference $t$, and $t$ is the average value of the image of the reference.

The process was carried out with normxcorr2 function in Matlab.[6] The calculated coefficients $Y(u, v)$ were recorded in the matrix. The coordinates of the maximum element of the matrix corresponded to the best match. The process of matching is presented in Figure 1. 
a)

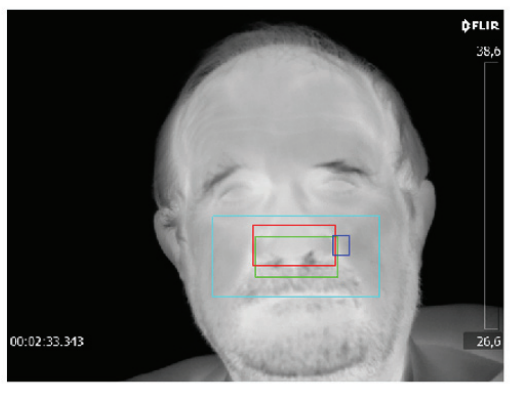

b)
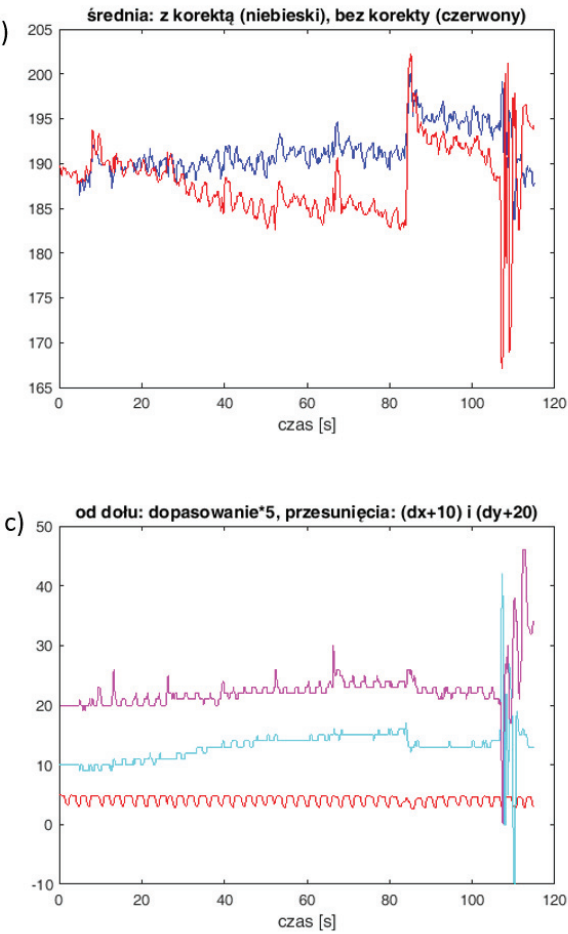

b) means: with correction (blue), without correction (red)

c) from the bottom: adjustment ${ }^{*}$, shifts: $(d x+10)$ and $(d x+20)$

Fig. 1. The process of area adjustment and correction of the position of the tracked object (as described in the text).

The following areas used in the algorithm are marked on the image presented in Figure 1a:

- The light blue border defines the area within which the reference is sought.

- The green border indicates the initial position of the nose - the area, which was adjusted in subsequent frames of the film

- The red border surrounds the best match.

- The dark blue border defines the studied area from which the average temperature, reflecting the mean value calculated for the pixels contained in the window, was calculated.

The course of temperature changes in the studied area is presented in Figure 1b. The red colour denotes mean values calculated for the pixels in the dark blue window that did not change its position. The blue chart is an analogous curve, accounting for the cor- 
rection of the position caused by movements of the face, with the data uploaded from the dark blue frame, whose position was strictly connected to the reference point of adjustment discovered (the red frame - Fig. 1a). The correction of location resulted in an observable change in the average temperature in the same area of the face, visible in the blue chart (Fig. 1b), which is substantiated by the horizontal character of the trend line in the blue chart.

Figure 1c presents additional curves illustrating the process of adjustment. The curves are shifted vertically or rescaled so as they did not superimpose. The bottom line presents the successive values of $Y(u, v$,$) adjustment coefficients, which were multiplied$ by five. The oscillations visible in the chart reflect the small changes in the successive pictures caused by the subject breathing through his nose. The two successive charts present the registered changes in the location of the face ("matching point") on axis $\mathrm{x}$ (light blue) and axis y (magenta), respectively. As can be seen from the chart, these are deviations in the range of several pixels (the vertical axis in the last two charts corresponds to distances in pixels.) More significant changes, visible towards the end of the test, are the result of quick intentional movements of the subject's head.

\section{Polygraph record versus the results of thermal visual detection}

The experiment in question was conducted with a FLIR A655sc infrared camera mounted on a tripod approximately $90 \mathrm{~cm}$ (35 in.) away from the face of the subject. Such a setting of the camera had the face of the subject fill most of the shot. The software provided by the producer allowed to record video stream at $640 \times 480$ pixel resolution, at 30 frames a second. Such clips were later converted to a format compatible with Matlab (version 2016b).

The video was recorded during a practical stimulating test preceding the routine polygraph examination,[7] during which the subject was presented with six questions (stimuli).

The video recording the changes of the image of the subject's face was analysed in three configurations presented in Figure 2. Like in the previous case, the green border surrounds the area of the nose used for the correlation-based recalculation of the minor shifts caused by the movements of the subject's head. An analysis of the course of blood vessels in the face made it possible to define the remaining areas (windows).[8] The red (filled) window served the detection of changes of the lips position. 
a)

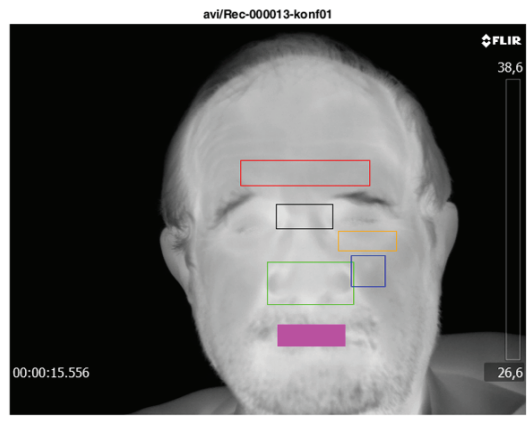

b)

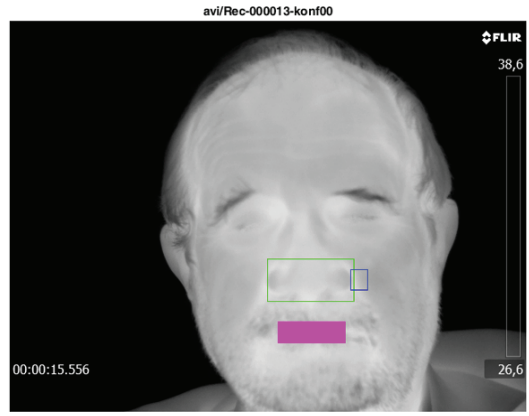

c)

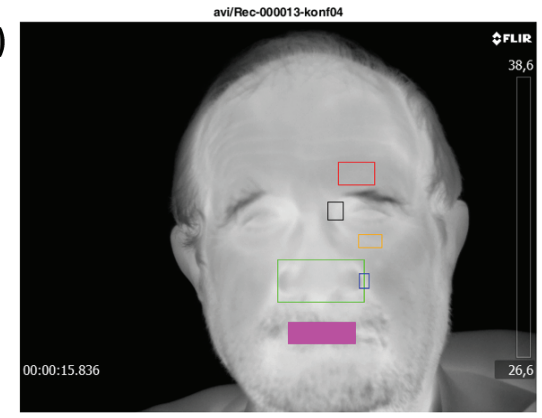

Fig. 2. Three examples of configuration: a), b), c).

Configuration "conf00" consisted of one medium-sized area situated around the nose (see: Fig. 2b). The remaining two ("conf01" and "conf04") make it possible to upload information from the areas of the forehead, base of the nose, cheek below the left eye, and cheek by the nose. Area selection was based on the three criteria discussed above. The selected areas differ in size: they are much larger in the case of "conf01" than of "conf04" (see: Figs. 2a and 2c).

Figure 3 presents a fragment of a polygraph recording with the registration result of a psychogalvanometer sensor recording changes in skin resistance of the subject (the galvanic skin response GSR, the electrodermal activity EDA), being part of the Lafayette LX4000 polygraph. 


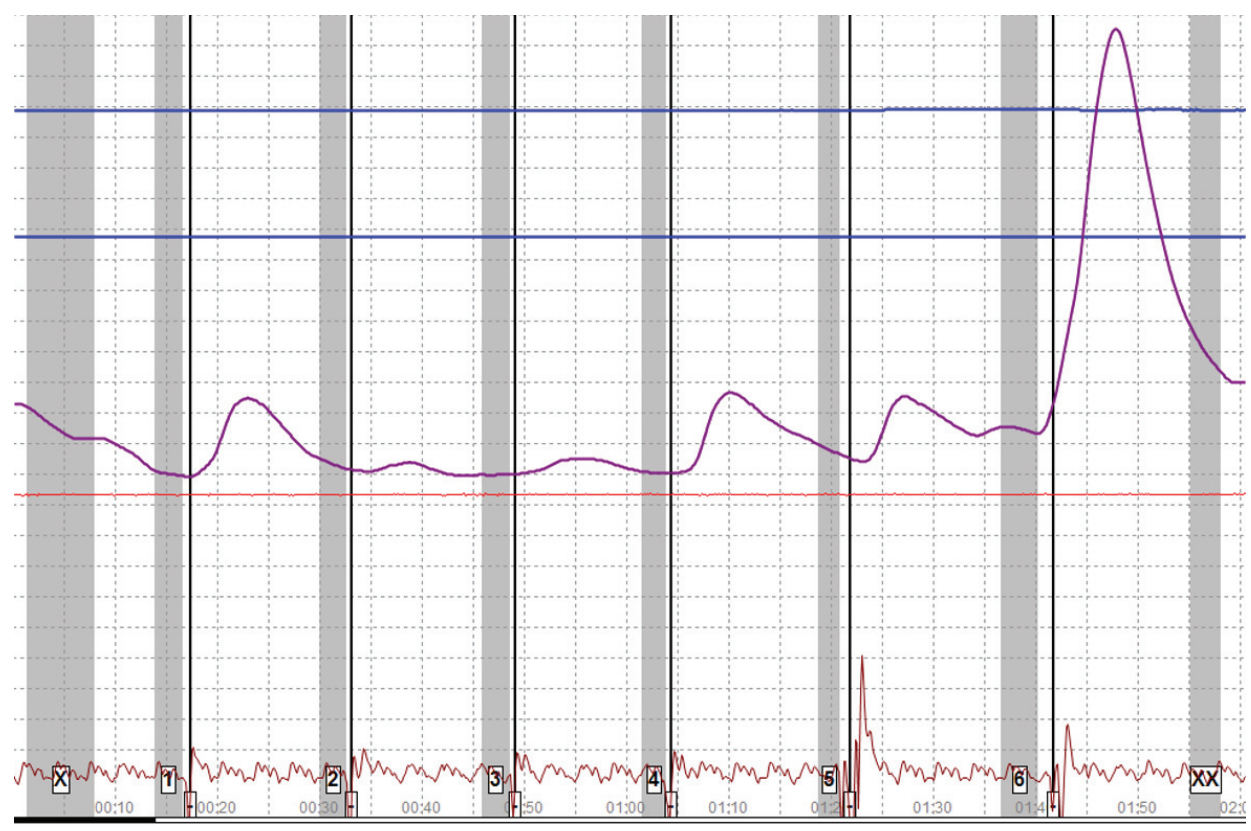

Fig. 3. Result of GSR sensor recording during a stimulation test.

Figure 4 presents the charts for the configurations discussed above. Each time a stimulus is presented is marked with colourful rectangles at the top of the figure. The green colour of the rectangle means a signal received on the GSR curve, whose counterpart will be sought on the infrared chart. The colours of the charts presented in the figure below correspond to the colours of the areas shown in Fig. 2. The configuration config00 is presented with dotted line, config01 - with thin lines, and config04 with bold lines.

The visual analysis conducted by assessment of the value of local maximums shows significant differences observed for various locations and various sizes of the areas. The listing for individual answers is provided in Table 1. 


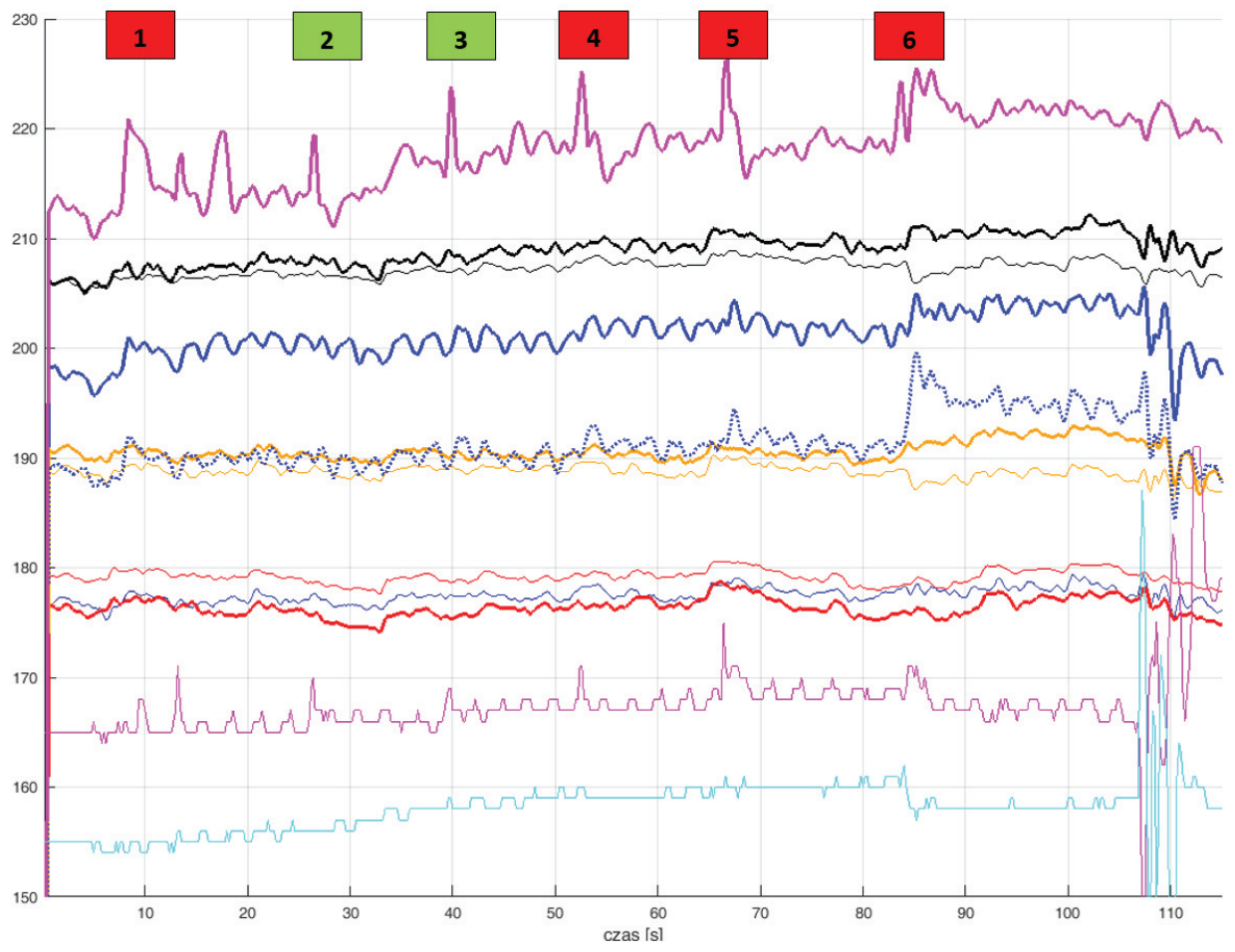

Fig. 4. Mean values calculated in windows defined for the following configurations: configOO - dotted line, config01 - thin lines, config04 - thick lines. Bottom: movements of the face in direction $x$ (light blue) and direction y (magenta). Top: the area of the mouth (thick magenta line). A size 3 averaging filter was used for the functions calculated in the windows. The numbered rectangles mark the times when the subject provided the answers. 


\begin{tabular}{|c|c|c|c|c|c|c|c|c|c|}
\hline \multirow{2}{*}{ Configuration } & \multirow{2}{*}{ Area } & \multirow{2}{*}{ Size } & \multicolumn{6}{|c|}{ Answer } & \multirow{2}{*}{$\begin{array}{c}\text { Comments / (General } \\
\text { Assessment }+ \text { or }-)\end{array}$} \\
\hline & & & 1 & 2 & 3 & 4 & 5 & 6 & \\
\hline conf01 & Forehead & big & + & + & - & - & + & + & \multirow{2}{*}{$\begin{array}{c}\text { Some responses are } \\
\text { delayed } /(-)\end{array}$} \\
\hline conf04 & Forehead & small & + & + & - & - & + & + & \\
\hline conf01 & \begin{tabular}{|c|} 
Base \\
of the nose
\end{tabular} & big & + & - & - & - & + & $-!$ & $\begin{array}{l}\text { minimum instead of the } \\
\text { maximum / (-!) }\end{array}$ \\
\hline conf0 4 & \begin{tabular}{|c|} 
Base \\
of the nose
\end{tabular} & small & + & - & - & - & + & + & $(-)$ \\
\hline conf01 & $\begin{array}{l}\text { Cheek } \\
\text { under } \\
\text { the eye }\end{array}$ & big & + & - & - & - & + & $-!$ & $\begin{array}{l}\text { minimum instead of the } \\
\text { maximum / (-!) }\end{array}$ \\
\hline conf0 4 & $\begin{array}{l}\text { Cheek } \\
\text { under } \\
\text { the eye }\end{array}$ & small & + & - & - & - & + & + & $(-)$ \\
\hline conf01 & \begin{tabular}{|c|} 
Cheek \\
by the nose
\end{tabular} & big & + & + & - & + & + & - & $(-)$ \\
\hline conf0 4 & \begin{tabular}{|c|} 
Cheek \\
by the nose
\end{tabular} & small & + & - & - & + & + & + & $(+)$ \\
\hline conf00 & \begin{tabular}{|c|} 
Cheek \\
by the nose
\end{tabular} & medium & + & - & - & + & + & + & $(+)$ \\
\hline
\end{tabular}

Table 1. Visual analysis of the defined areas: presence of local maximums.

As shown in Fig. 4 and Table 1, the lines for large and small areas are consistent, yet they do not make it possible to discriminate the reactions consistent with the GSR recording. Irregular situations are marked with exclamation marks. Characteristically, amplitudes of the functions obtained are lower for larger areas. Full consistency was obtained for the areas presented in the last two rows of Table 1.

\section{Impact of minor changes of window location and size on the quality of the recording}

Fig. 5 presents configuration config05 used for the analysis. The reference area was the window defined in the configuration config00 (see: the last line in Table 1 and Fig. 2b). The following investigated areas were moved (in respect to the reference area) towards the forehead of the subject, and their size was changed (see: Fig. 5). 
a)

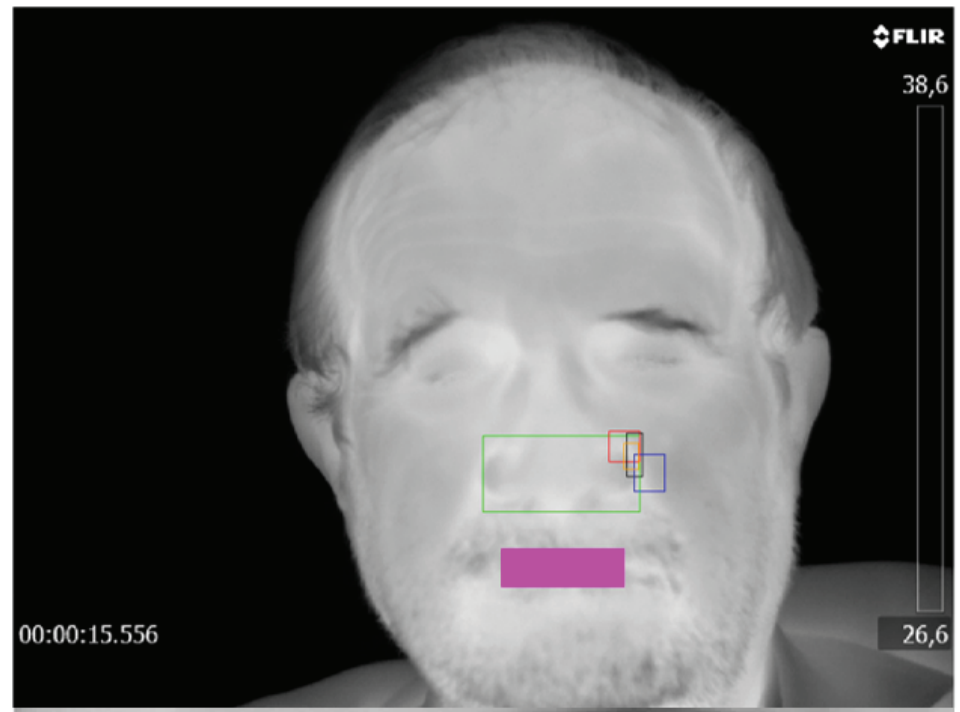

b)

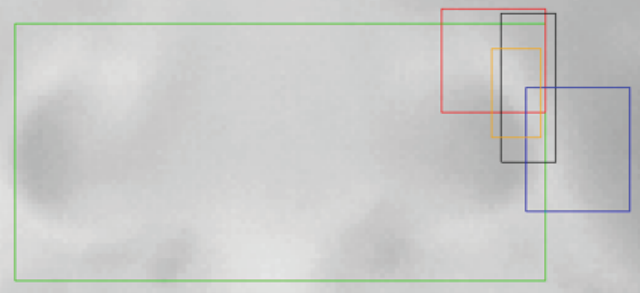

Fig. 5 a) Overview of config05 configuration, b) enlarged part of the image showing the defined areas.

Figure 6 gathers the functions (charts, curves) obtained, as previously, by averaging pixel values in individual areas. Five functions (counting from the top) are presented in "raw" form, which means that they were not subjected to averaging filtration. The order of presentation of the functions on the vertical axis corresponds to the successive 
locations of the bottom edges of the areas defined in the config0 5 configuration, and presented in Fig. 5.

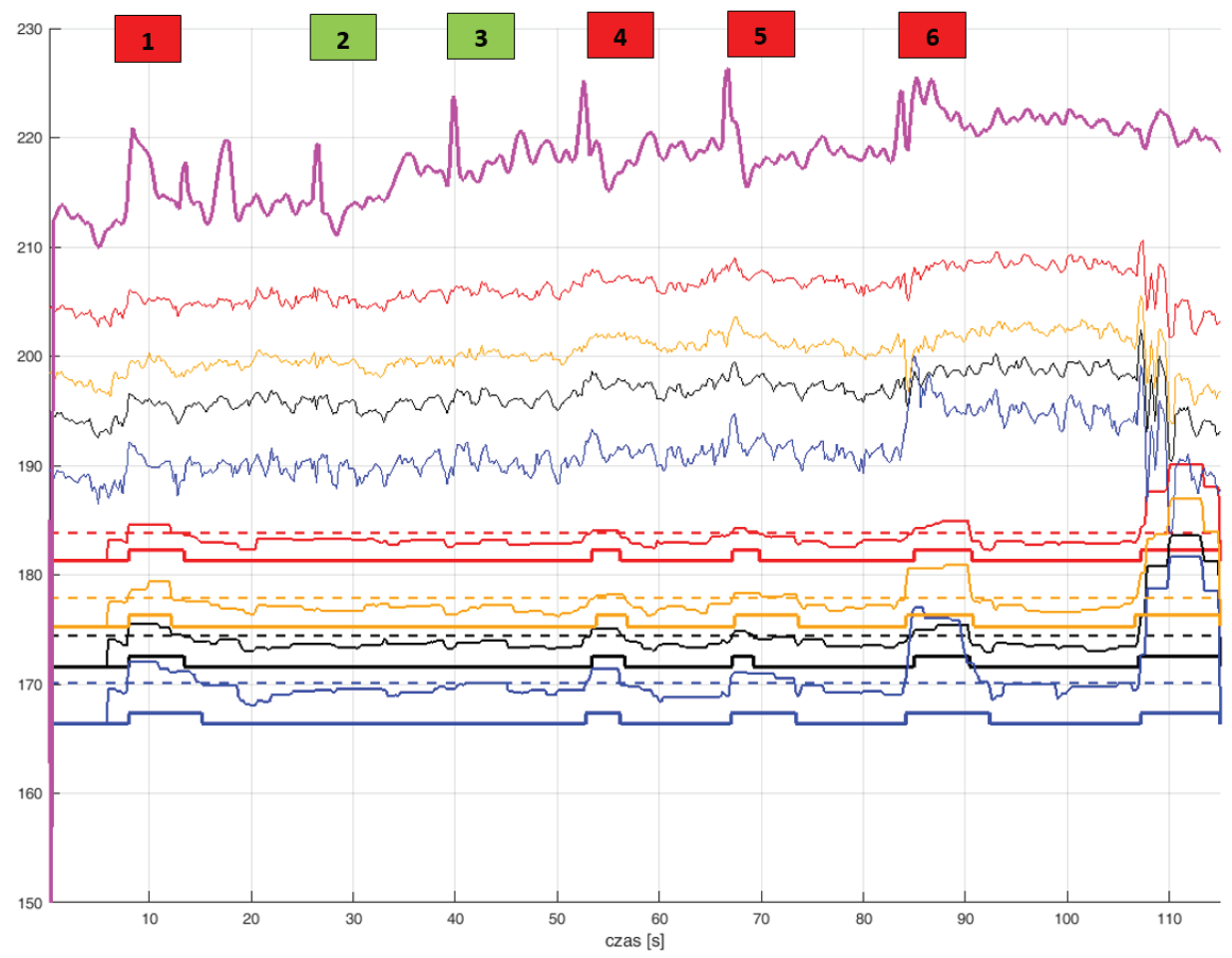

Fig. 6. Top to bottom: the function corresponding to the area "lips", with four functions calculated in the areas of configuration config05 (without the averaging filtration) below; further: results of detection and binarization of local maxima for four functions (described in the text).

Despite the differences in the location of the areas and their sizes, the functions have similar shape. The only significant differences were present around the 85 th second of the recording.

Detection of the maximum values corresponding to the subject's deceptive answers (GSR local maxima) is a difficult task in the case of thus presented charts. That is why the decision to develop the algorithm supporting the analysis was reached. The amplitude was computed in a window moving along the given function. It was calculated by the subtracting of the minimum value from the maximum value. The functions resulting from that calculation (curves) are presented in the bottom section of Fig. 6 (thin lines, situated from 165 to 185 on the vertical axis). 
This method made it possible to give emphasis to the local maxima. This made it possible to introduce cut-off lines (or thresholds, marked in broken lines in Fig. 6) precisely indicating the maxima, and consequently detecting the impact of temperature change caused by changes of emotions in the subject.

The position of the lines localising the maximums (thresholds) can be determined manually on the charts. Nonetheless, an attempt to generate them automatically was made, and in most cases provided successful results. To achieve this three methods were tested: 1) Definition of a threshold (a cut-off line localising the maxima) based on statistical analysis. To achieve that, the average and standard deviation were calculated for each of the four functions. The threshold was set at the level of mean value increased by half of standard deviation. Unfortunately, the results were unsatisfactory.

2) threshold level calculation based on the histogram of amplitudes of local functions, with the assumption that the histogram has a minimum that separates the answers during which the subject reacted from the ones during which the subject did not react (bi-modal histogram). The histograms for individual areas are presented in Fig. 7a (left column). However, the bi-modal assumption failed.

3) Using the standard histogram to calculate the accumulated histogram (see: Fig. 7b). After double "smoothing" (i.e. application of averaging filtration) with a window whose length is equal to 3 histogram bins (histogram bars), the difference (derivative) was calculated in a window with the length of 3 bins, and the maximum of the latter function was calculated (which correspond to the inflection point of the function of accumulated histogram). The threshold was situated in the histogram bin subsequent directly to the maximum (see: red curves and triangles on the $\mathrm{x}$-axis in Fig. $7 \mathrm{~b}$ ).

The third method turned out the best results, which are presented in Fig. 6 (see: the thick lines situated below the corresponding functions of "local amplitudes"). Table 2 contains the parameters calculated for individual areas.

\begin{tabular}{|c|c|c|c|c|c|c|c|c|c|}
\hline \multirow{2}{*}{$\begin{array}{c}\text { Area } \\
\text { border } \\
\text { colour }\end{array}$} & \multirow{2}{*}{$\begin{array}{c}\text { Area field } \\
\text { (in pixels) }\end{array}$} & $\begin{array}{c}\text { Amplitudes of } \\
\text { "area average" } \\
\text { functions }\end{array}$ & $\begin{array}{c}\text { Amplitudes of } \\
\text { "local amplitudes" } \\
\text { functions }\end{array}$ & \multicolumn{4}{|c|}{$\begin{array}{c}\text { Parameters of "local } \\
\text { amplitudes" functions }\end{array}$} \\
\cline { 3 - 11 } & $\mathbf{0 \div 8 0 [ \mathbf { s } ]}$ & $\mathbf{0} \div \mathbf{1 0 0}[\mathbf{s}]$ & $\mathbf{0} \div \mathbf{8 0}[\mathbf{s}]$ & $\mathbf{0} \div \mathbf{1 0 0}[\mathbf{s}]$ & avg & std & $\begin{array}{c}\text { thresh } \\
\text { (meth. 3) }\end{array}$ & $\Delta p$ \\
\hline red & 441 & 6.3 & 6.9 & 3.3 & 3.7 & 1.9 & 0.75 & 2.6 & 0.76 \\
\hline orange & 180 & 7.3 & 7.3 & 4.1 & 5.6 & 2.1 & 1.15 & 2.6 & 0.76 \\
\hline black & 330 & 6.9 & 7.7 & 4.0 & 4.0 & 2.2 & 0.83 & 2.8 & 0.7 \\
\hline blue & 525 & 8.2 & 13.6 & 5.7 & 10.7 & 3.6 & 1.88 & 3.8 & 0.87 \\
\hline
\end{tabular}

Table 2. Parameters of the areas defined in configuration config05. 


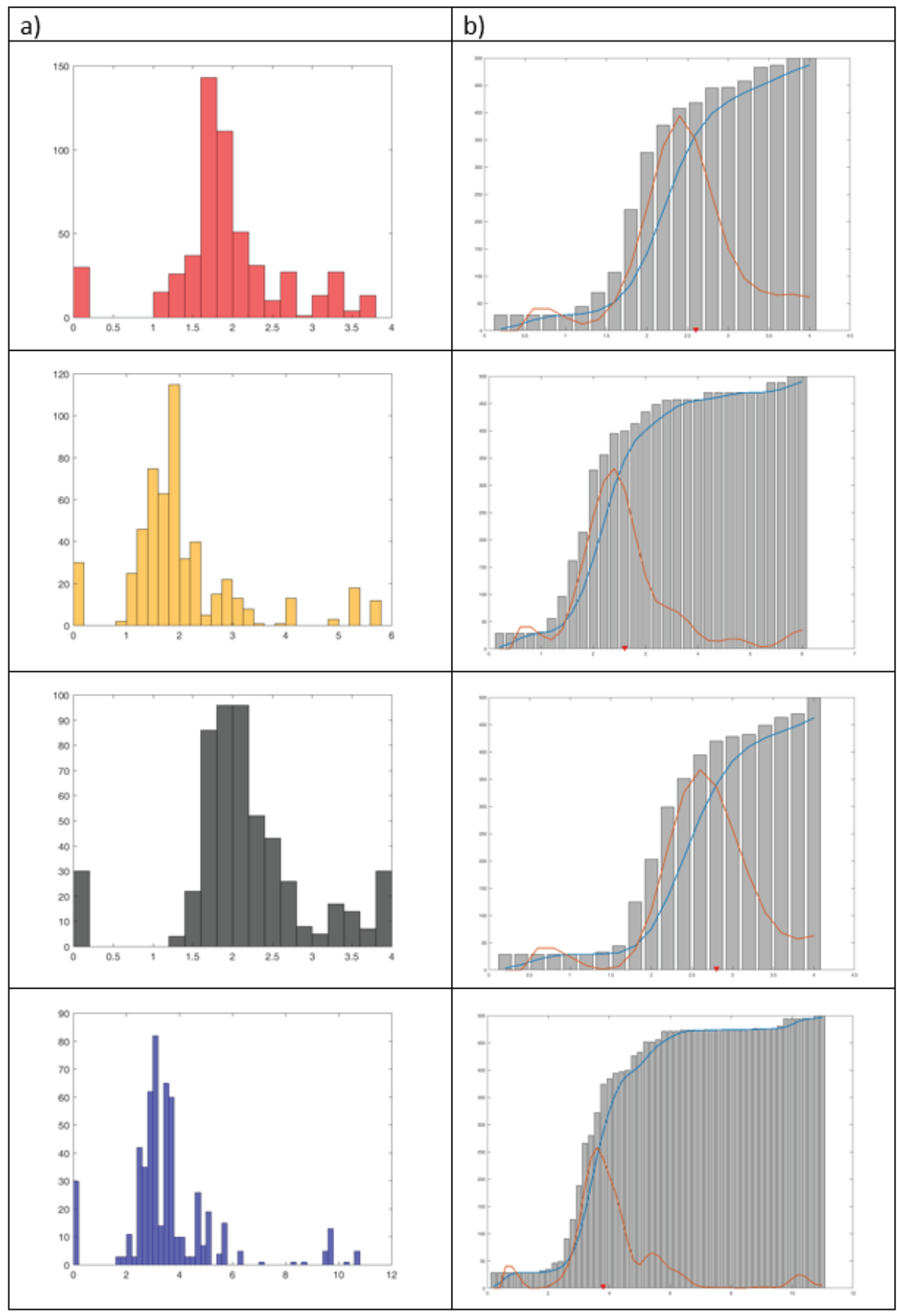

Fig. 7. a) histograms of the functions of "local amplitudes", b) cumulated histograms corresponding to the above, with smoothed functions (blue), derivatives (red), and thresholds (little red triangles). 
Irregularities in the function of the means calculated in the tracked areas were recorded in the 85th second. For that reason, amplitudes were calculated for two intervals: [0, $80]$ and $[0,100]$. The average (avg) and the standard deviation $(s t d)$ were calculated for the scope $[1,100]$. The threshold calculated with the third method (see above) does not depend on the time interval. The $D p$ parameter defined manually (see: Fig. 8 ) is thought-provoking.

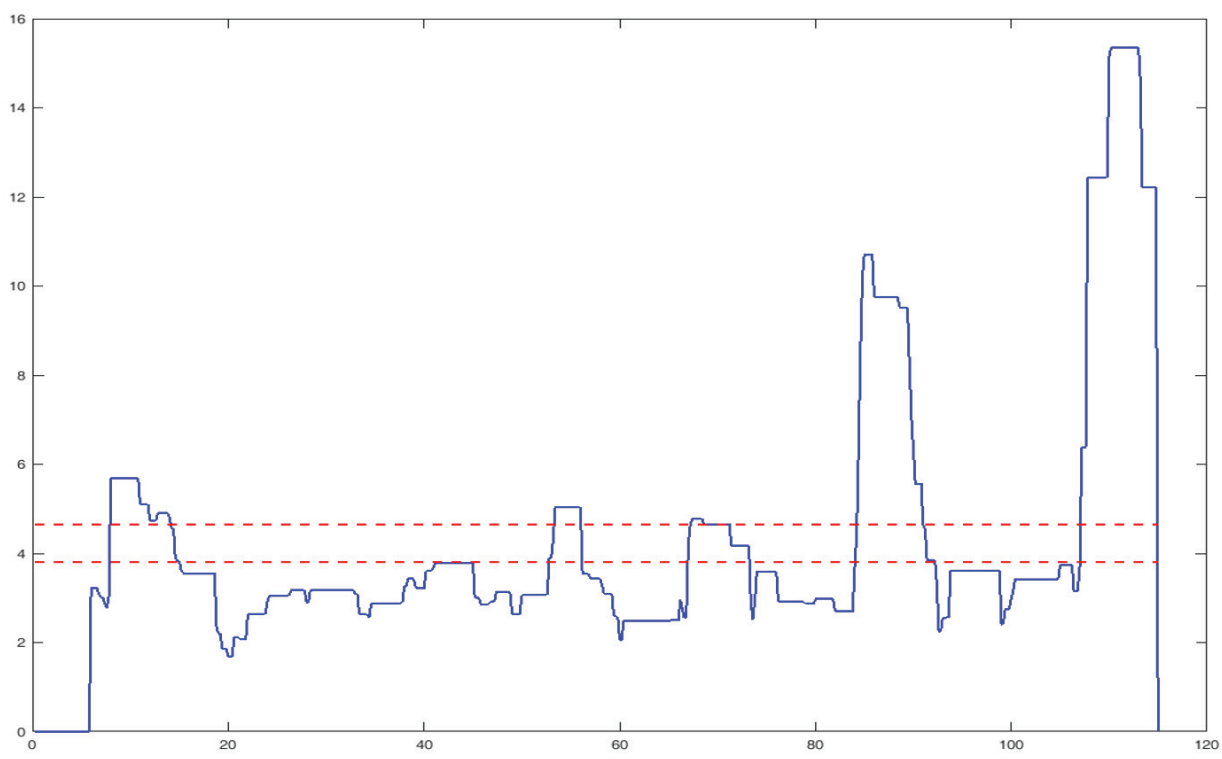

Fig. 8. The scope of changes for the correctly differentiating Dp threshold for the "blue" area.

This is a threshold variability range that correctly separates the answers during which a reaction occurred and was registered by the psychogalvanometer (GSR) from the ones that elicited no such reaction. It is the highest for the "blue" area, which also towers above the remaining parameters, when calculated both for the functions of "averages from the area" and of "local amplitudes". As the comparison of parameters demonstrates, it is mostly the location of the "window" that matters. Its size (area) is of less importance, although too small windows should not be defined, see: the second column of Table 2. The size of standard deviation outside the "blue" area does not influence the $D p$ parameter. 


\section{Analysis of the records obtained while performing Utah ZC test}

The following stage of the study consisted in conducting a standard diagnostic polygraph test, during which the subject is seated in a special armchair, and instructed not to move but only provide short "yes" and "no" answers to the questions asked. The study conducted concerned a simulated situation in experimental conditions. Let us only explain that the task of the subject was to conceal the fact that the subject had visited a shooting range and fired shots at an image of a woman on a poster.

The polygraph examination made use of the diagnostic test that belongs to the routinely used Utah Zone Comparison Test (Utah ZCT, UZCT). The test was performed in single-question version. Control questions on the so-called "focused lie" and the following critical questions were used:

- Was it you who shot at the image of a woman?

- Did you have a gun in your hand on that day?

- Did you shoot at a shooting range on that day?

Thanks to the application of the standards of a polygraph examination, facial temperature changes could be examined for multiple stimuli coming in the form of questions in polygraph tests.[7] The examination was composed of a stimulation test ("with a number"), and 3 or 5 diagnostic tests, each of which contained three critical and three control questions.

To increase the comfort of the subjects, the recording was made from a camera set approximately $2 \mathrm{~m}$ (78 in.) away from the face of the subject, and not just $90 \mathrm{~cm}(35 \mathrm{in}$.) away as previously. Fig. 9 shows a sample configuration of the scene for this phase of the experiment. Polygraph sensors are attached to the body of the subject and "windows" can be seen delineated on the image of the subject's face. 


\section{JUTAH 5-000025-ramka-125-konf00}

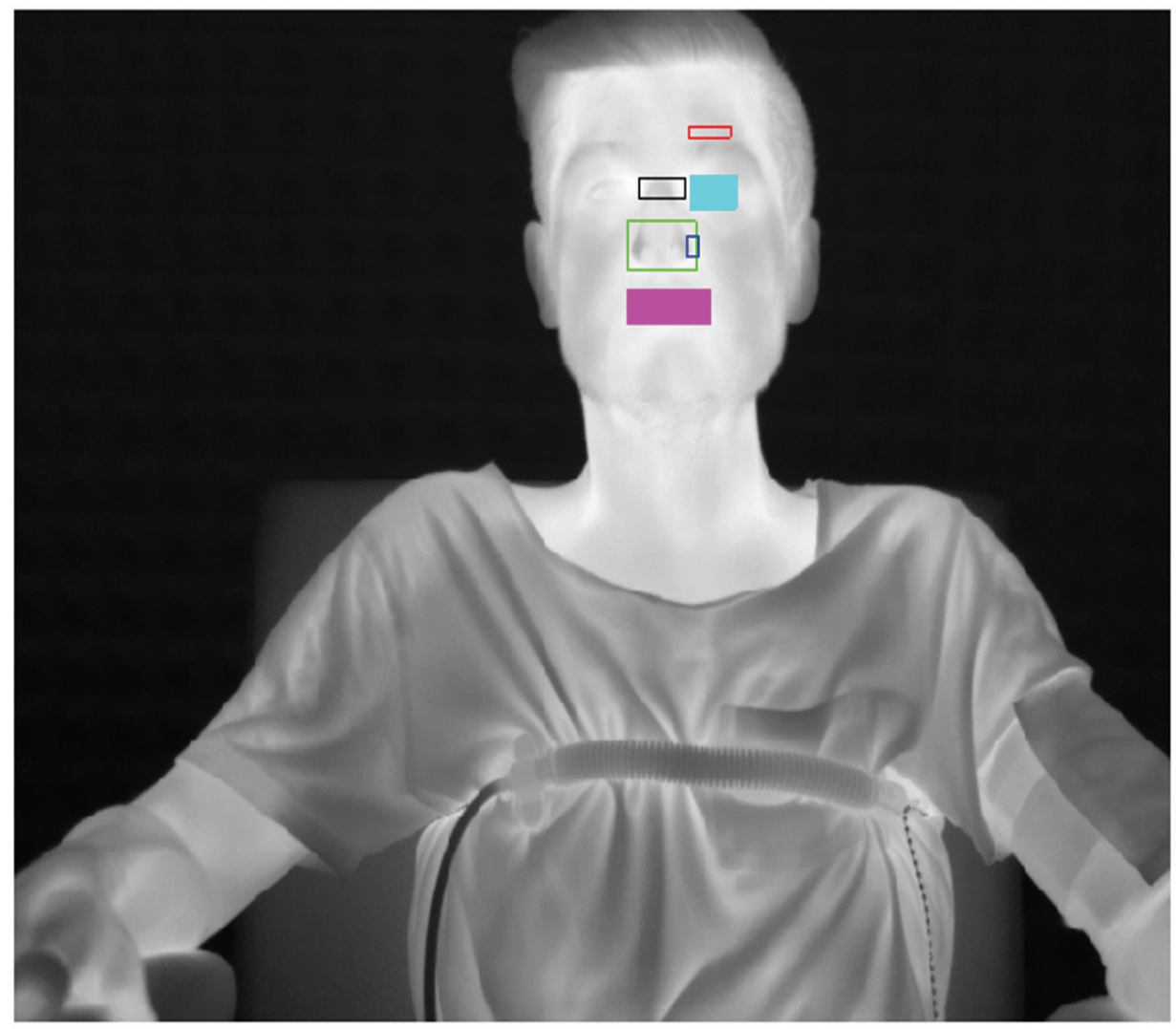

Fig. 9. Configuration defined for the subject; a similar configuration was used in the remaining experiments.

As the illustration clearly shows, moving the infrared camera to distance of around $2 \mathrm{~m}$ away from the face of the subject radically reduced the image of the face, and consequently the area of the "windows" to be analysed.

Figures 10, 11, and 12 present three sample recordings of changes of facial temperature registered in parallel with the polygraph examination. To offset partially the reduction of the sizes of the "windows", the analysis was conducted on 16-bit pixel representation images (see: axis y in Fig. 10, 11, and 12). 


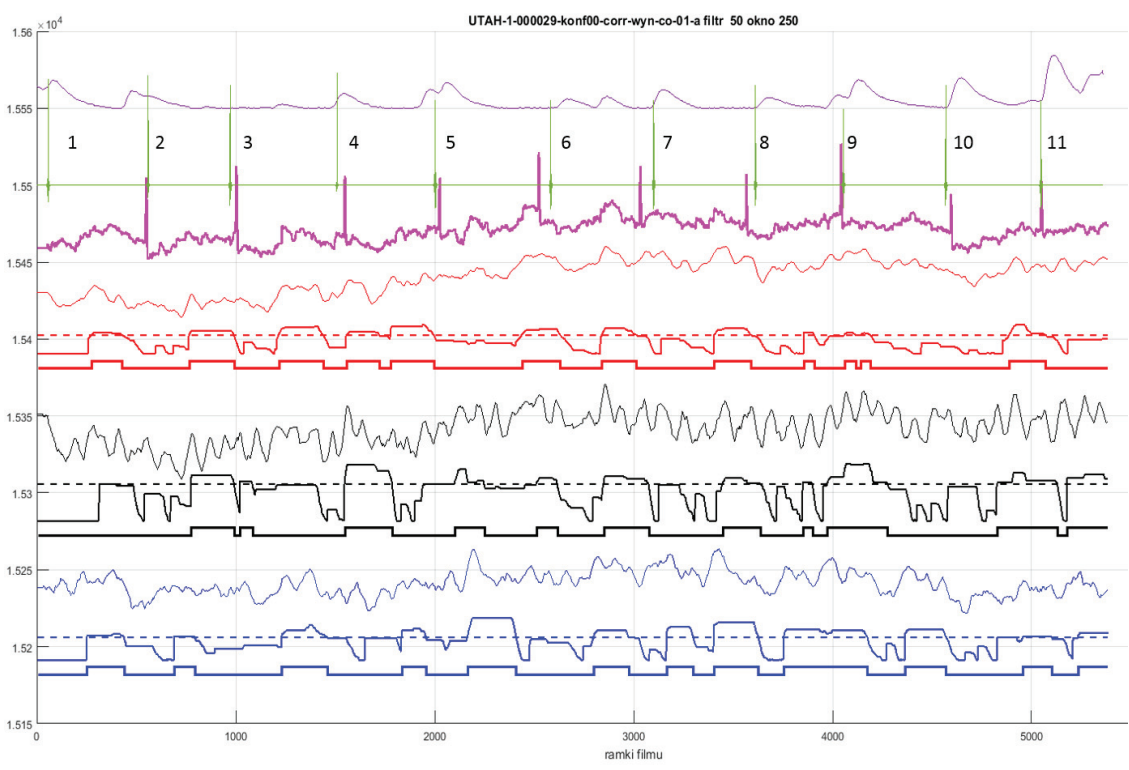

Fig. 10. Results of analysis of the video stream from the infrared camera for person AC144. Colours in line with configuration described in Fig. 9.

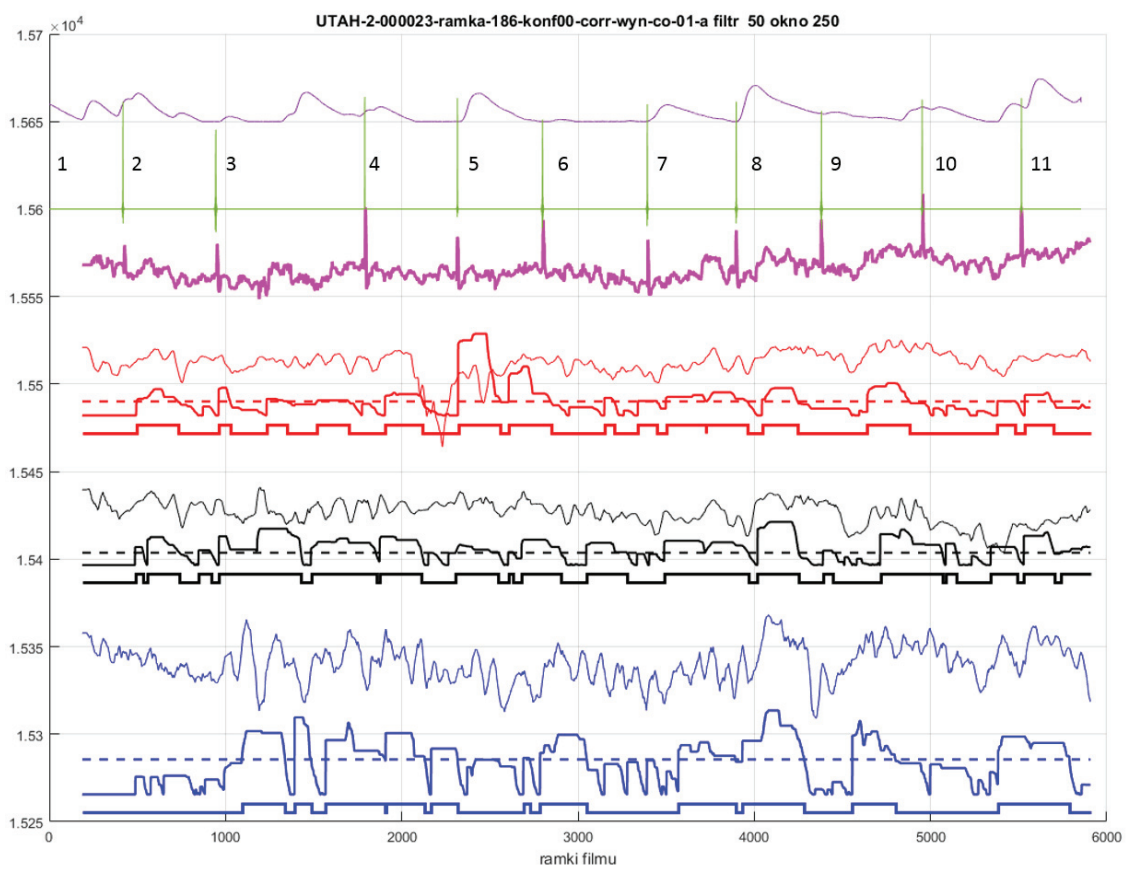

Fig. 11. Results of analysis of the video stream from the infrared camera for person KT 100, series 2. Colours in line with configuration described in Fig. 9. 


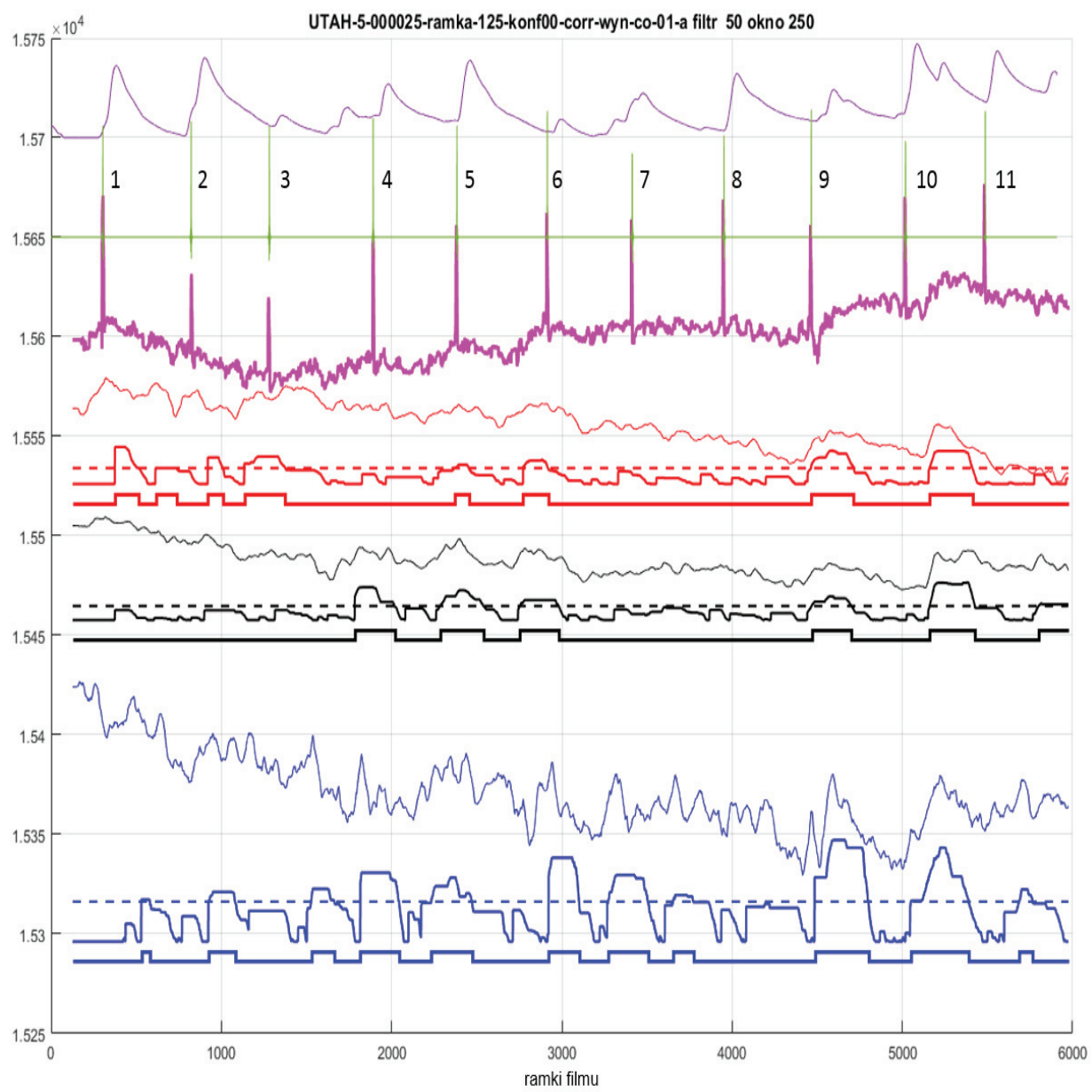

Fig. 12. Results of analysis of the video stream from the infrared camera for person KT 100 series 5. Colours in line with configuration described in Fig. 9.

The thresholds (situation of the lines detecting maxima) were set manually. Each recording was complemented with two charts visible at the top of the figures 10,11 , and 12 . Violet colour denotes the signal from the psychogalvanometer (one of polygraph sensors recording the galvanic skin response GSR), The green colour denotes the moments (recorded by the asker) when answers were given. Numbers of the questions asked were entered between the curves.

The data in Table 3 is based on the readings from the polygraph and infrared recordings presented in figures 10,11, and 12. Due to the shape of the curves, it is best to conduct 
comparative analyses by comparing infrared signals (the chart of facial temperature changes) with the signal from the psychogalvanometer sensor (the curve of galvanic skin response changes, GSR). The latter is presented in figures 10,11, and 12 as the first curve from the top (violet).

\begin{tabular}{|c|c|c|c|c|c|c|c|c|c|c|c|c|c|}
\hline \multirow{2}{*}{$\begin{array}{l}\text { FILM } \\
.000029\end{array}$} & \multicolumn{13}{|l|}{ Probant } \\
\hline & AC144 & pytanie nr & INT1 & S2 & N3 & C4 & R5 & N6 & C7 & R8 & N9 & C10 & R11 \\
\hline \multirow[t]{6}{*}{ Diagram 1} & \multirow{3}{*}{ POLIGRAF } & Pneumo & $\mathrm{Rm}$ & & & & & & & & & & \\
\hline & & GSR & & & & $\mathrm{Rm}$ & $\mathrm{Rm}$ & $\mathrm{Rd}$ & & $\mathrm{Rd}$ & & & \\
\hline & & CARDIO & $\mathrm{Rm}$ & & & $\mathrm{Rd}$ & & & & & & Rd & \\
\hline & \multirow{3}{*}{ Termowizja } & czoło & & & & $\mathrm{x}$ & & & & & $\mathrm{x}$ & & \\
\hline & & oczy & & $\mathrm{x}$ & & $\mathrm{x}$ & & & $\mathrm{x}$ & & $\mathrm{x}$ & & \\
\hline & & nos & & & & & $\mathrm{x}$ & & $\mathrm{x}$ & & $\mathrm{x}$ & & \\
\hline FILM & \multicolumn{13}{|l|}{ Probant } \\
\hline \multirow{7}{*}{$\begin{array}{l}.000023 \\
\text { Diagram } 2\end{array}$} & KT100 & pytanie nr & INT1 & $S 2$ & N3 & C4 & R5 & N6 & C7 & R8 & N9 & $\mathrm{C} 10$ & R11 \\
\hline & \multirow{3}{*}{ POLIGRAF } & Pneumo & & & & & & & & & & & \\
\hline & & GSR & & & & & $\mathrm{Rm}$ & & & $\mathrm{Rd}$ & & & $\mathrm{Rd}$ \\
\hline & & CARDIO & & $\mathrm{Rm}$ & & $\mathrm{Rm}$ & & & $\mathrm{Rd}$ & & & & $\mathrm{Rm}$ \\
\hline & \multirow{3}{*}{ Termowizja } & czoło & & & $\mathrm{x}$ & $\mathrm{x}$ & & & & $\mathrm{x}$ & & & \\
\hline & & oczy & & & & $\mathrm{x}$ & $\mathrm{x}$ & & & $\mathrm{x}$ & & & \\
\hline & & nos & & $\mathrm{x}$ & $\mathrm{x}$ & & & & & $\mathrm{x}$ & & & $\mathrm{x}$ \\
\hline \multirow{2}{*}{$\begin{array}{l}\text { FILM } \\
.000025\end{array}$} & \multicolumn{13}{|l|}{ Probant } \\
\hline & KT100 & pytanie nr & INT1 & S2 & N3 & $\mathrm{C} 4$ & R5 & N6 & C7 & R8 & N9 & $\mathrm{C} 10$ & R11 \\
\hline \multirow[t]{6}{*}{ Diagram 3} & \multirow{3}{*}{ POLIGRAF } & Pneumo & & & & & & & & & & & \\
\hline & & GSR & & $\mathrm{Rm}$ & & & $\mathrm{Rm}$ & & $\mathrm{Rd}$ & & & & $\mathrm{Rd}$ \\
\hline & & CARDIO & & $\mathrm{Rm}$ & & & $\mathrm{Rm}$ & & $\mathrm{Rm}$ & & & & $\mathrm{Rd}$ \\
\hline & \multirow{3}{*}{ Termowizja } & czoło & & & $\mathrm{x}$ & & & & & & & $\mathrm{x}$ & \\
\hline & & oczy & & & & & $\mathrm{x}$ & & & & & $\mathrm{x}$ & \\
\hline & & nos & & & & & & & & & $\mathrm{x}$ & $\mathrm{x}$ & \\
\hline
\end{tabular}

\section{Legend:}

$\mathrm{X}$ - the line of temperature rises in the given window, after the start of the question $\mathrm{Rm}$ - minor reaction on polygraph channel

$\mathrm{Rd}$ - major reaction on polygraph channel

Table 3. Comparison of the results of detection of facial temperature changes and the reactions recorded by the polygraph. 
A decision was made to assess globally a whole polygram (a single test) for the needs of the table, marking the strongest $(\mathrm{Rd})$ and two successive $(\mathrm{Rm})$ reactions. Only the volume amplitude was considered a reaction on cardio and GSR channels, as their extensity and duration were disregarded. The reactions to some questions on cardio and GSR channels were similar in volume, in which case they were appropriately labelled; if two reactions were present on one channel during a test, both received the Rd label.

\section{Summary}

The purpose of the experiment was first to find an independent method for transforming infrared camera image into a chart (curve), secondly to develop a method that would allow continuous observation and registration of the subject's facial temperature changes, and thirdly to find an answer to the question whether human emotional reactions registered with a standard computer polygraph (Lafayette, LX-4000) have their counterparts in the changes of face temperature recorded with infrared camera (FLIR A655sc).

The method of transforming infrared camera image into a curve described in literature [4] was successfully used and improved.

A number of characteristic areas of the face were selected and studied to analyse the infrared test video stream recorded at a close distance (approx. $90 \mathrm{~cm}$ ). An algorithm detecting significant changes in the temperature of the selected areas was created. Moreover, areas whose analysis offers best results were defined. Full consistency with GSR recording was obtained for two areas situated near the nose (see: Table 1, the last two rows).

The comparison of polygraph test recordings and thermal reactions, described in section 5, only partially validates the claim that the reactions are consistent (parallel, simultaneous). The curves obtained by the averaging of pixel values from individual areas seem consistent with one another, yet are only to a small degree consistent with the registered skin galvanic response GSR (see: figures 10, 11 and 12, and Table 3). This, however, does not mean that no consistency was discovered between the obtained values, as such consistencies were e.g. observed for the questions N9 and C10 (for video stream 000025).

What seems to be the reason for deterioration of infrared detection in the experiment described in section 5 above, was the excessive distance between the camera and the face of the subject. The reason for such results may also be the excessively small size of the sample used for the study. Theoretically, with a far larger sample (the larger group 
of persons examined), the consistency of reactions could be greater. Further tests must first be performed on a larger sample, and secondly the infrared camera must be situated closer to the face (e.g., at a distance of $90 \mathrm{~cm}$ ). Moreover, the claim made in literature[9] that the galvanic skin response may precede other, notably thermal, reactions in time also requires validation through experiments.

\section{References}

[1] Gołaszewski M., Zając P., Widacki J. (2015), Thermal Vision as a Method of Detection of Deception, European Polygraph, 9, 1, p. 31.

[2] US Patent 6996256, patent EP 1286620 (Detection system and method using thermal image analysis, 2006).

[3] Patent EP 0885587 (Thermal imaging method and apparatus, 1998).

[4] Widacki J., Widacki M., Antos J. (2016), Preparation to Experimental Testing of the Potential from Using Face Temperature Changes Registered with an Infrared Camera in Lie Detection, European Polygraph, 10, 1, p. 35.

[5] Matlab release 2016b, The MathWorks, Inc. Natick, Massachusetts.

[6] Lewis J.P. (1995), Fast Template Matching, Vision Interface, p. 120-123.

[7] Kryminalistyka, ed. by J. Widacki (2016), 3rd ed., CH Beck, Warszawa.

[8] Aleksandrowicz R. (2009), Maty atlas anatomiczny, PZWL, Warszawa, p. 97.

[9] Boucsein W. (2012), Electrodermal Activity, 2nd ed., Springer, New YorkDordrecht-Heidelberg- London, p. 29-36, 130 and ff. 